\title{
Efficacité et innocuité des agents antiviraux contre le SRAS-CoV-2, selon des données d'études sur la COVID-19 et d'autres infections virales aiguës : revue systématique et méta-analyse
}

Wei Liu PhD, Pengxiang Zhou MPharm, Ken Chen MPharm, Zhikang Ye MPharm, Fang Liu MSc, Xiaotong Li MSc, Na He MSc, Ziyang Wu BSc, Qi Zhang MSc, Xuepeng Gong PhD, Qiyu Tang BSc, Xin Du BSc, Yingqiu Ying MSc, Xiaohan Xu MSc, Yahui Zhang BSc, Jinyu Liu PhD, Yun Li MSc, Ning Shen MD, Rachel J. Couban MA, Quazi I. Ibrahim MSc, Gordon Guyatt MD MSc, Suodi Zhai BSc

Citation : CMAJ 2020 July 6;192:E734-44. doi : 10.1503/cmaj.200647-f; diffusion hâtive le 3 juin 2020

Voir l'article connexe en anglais ici : www.cmaj.ca/lookup/doi/10.1503/cmaj.200648; voir l'article connexe en français ici : www.cmaj. ca/lookup/doi/10.1503/cmaj.200648-f

\section{RÉSUMÉ}

CONTEXTE : On donne de façon empirique des agents antiviraux à certains patients atteints de la maladie à coronavirus 2019 (COVID-19). Dans le but d'appuyer la rédaction de lignes directrices sur la prise en charge de la COVID-19, nous avons réalisé une revue systématique des bénéfices et des préjudices associés à 7 traitements antiviraux contre cette infection.

MÉTHODES : Nous avons effectué des recherches dans MEDLINE, Embase, le Cochrane Central Register of Controlled Trials (CENTRAL), PubMed et 3 bases de données chinoises (CNKI, Wanfang Data et SinoMed) jusqu'au 19 avril 2020, dans medRxiv et ChinaXiv jusqu'au 27 avril 2020, ainsi que dans Chongqing VIP jusqu'au 30 avril 2020. Nous avons sélectionné des études sur la ribavirine, la chloroquine, l'hydroxychloroquine, l'umifénovir (Arbidol), le favipiravir, l'interféron et le lopinavir/ritonavir. Lorsqu'il n'y avait pas de données directes d'études sur la COVID-19, nous avons retenu des données indirectes d'études sur le syndrome respiratoire aigu sévère (SRAS) et le syndrome respiratoire du Moyen-Orient (SRMO) pour l'analyse de l'efficacité, et d'études sur d'autres infections respiratoires virales aiguës pour l'analyse de l'innocuité.

RÉSULTATS : Le taux de décès chez les patients atteints d'une forme sans signe clinique de gravité de COVID-19 était extrêmement bas, ce qui ne permet pas de conclure à un effet important sur la mortalité. Nous n'avons obtenu que des données de très faible qualité indiquant que la plupart des traitements avaient peu ou pas de bénéfices sur les paramètres à l'étude, quelle que soit la gravité de la COVID-19. Seule exception: le traitement au lopinavir/ritonavir, pour lequel nous avons obtenu des données de faible qualité faisant état d'une réduction de la durée du séjour en unité de soins intensifs (différence des risques [DR] 5 jours de moins, intervalle de confiance [IC] de $95 \% 0$ à 9 jours) et de la durée d'hospitalisation (DR 1 jour de moins, IC de $95 \% 0$ à 2 jours). En ce qui concerne l'innocuité, les données étaient de faible ou de très faible qualité, sauf pour le traitement au lopinavir/ ritonavir, où des données de qualité moyenne laissaient supposer une augmentation probable de la diarrhée, des nausées et des vomissements.

INTERPRÉTATION : À l'heure actuelle, rien ne prouve de façon convaincante que les traitements antiviraux apportent des bénéfices importants dans la lutte contre la COVID-19, bien que les données propres à chaque traitement n'excluent pas cette possibilité. D'autres essais randomisés et contrôlés menés auprès de patients atteints de la COVID-19 sont nécessaires avant de pouvoir recourir à ces traitements en toute confiance.

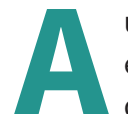
u 30 avril 2020, la maladie à coronavirus 2019 (COVID-19) ${ }^{1}$ était à l'origine de plus de 3 millions de cas d'infection et de plus de 210000 décès dans le monde². L'Organisation mondiale de la Santé (OMS) avait déjà déclaré que nous faisions face à une pandémie. L'agent pathogène, le coronavirus du syndrome respiratoire aigu sévère 2 (SRAS-CoV-2) ${ }^{3}$, est un nouveau bétacoronavirus présentant une ressemblance phylogénétique au SRAS-CoV (environ 79\%), ainsi qu'au MERS-CoV (environ 50\%), qui cause le syndrome respiratoire du Moyen-Orient ${ }^{4,5}$.

De nombreux essais sur des traitements potentiels contre la COVID-19 sont en cours, mais les stratégies thérapeutiques actuelles sont largement fondées sur des études précliniques et 
sur l'expérience acquise dans la lutte contre le SRAS et le $\mathrm{SRMO}^{6}$. Des cliniciens ont d'ailleurs administré un certain nombre de traitements antiviraux à des patients atteints de la COVID-197. La prise de décisions optimale quant à ces traitements nécessite des analyses systématiques des meilleures données probantes disponibles sur les antiviraux.

Nous avons mené une revue systématique afin d'appuyer la rédaction de lignes directrices formulant des recommandations sur les agents antiviraux (ribavirine, chloroquine, hydroxychloroquine, umifénovir, favipiravir, interféron et lopinavir/ritonavir) actuellement utilisés pour traiter la COVID-198 ${ }^{8}$ Comme le remdésivir n'était pas accessible au moment où la portée des lignes directrices a été établie, il n’a pas été compris dans la présente revue; toutefois, les résultats des premiers essais randomisés et contrôlés (ERC) sur ce médicament sont maintenant disponibles ${ }^{9}$. Notre revue s'intéresse à des ERC et à des études d'observation menés auprès de patients atteints de la COVID-19, du SRAS, du SRMO et de l'influenza.

\section{Méthodes}

\section{Conception de l'étude et sources de données}

Nous avons suivi le modèle PRISMA (Preferred Reporting Items for Systematic Reviews and Meta-Analysis). L'annexe 1 (accessible au www.cmaj.ca/lookup/doi/10.1503/cmaj.200647/tab-related -content) présente le protocole de la revue systématique, et l'annexe 2 (accessible au www.cmaj.ca/lookup/doi/10.1503/ cmaj.200647/tab-related-content), la stratégie utilisée pour nos 2 recherches indépendantes. Nous avons interrogé MEDLINE, Embase, le Cochrane Central Register of Controlled Trials (CENTRAL), PubMed et 3 bases de données chinoises (China National Knowledge Infrastructure [CNKI], Wanfang Data et SinoMed) jusqu'au 19 avril 2020, ainsi que medRxiv et Chinaxiv pour des prépublications jusqu'au 27 avril 2020. Nous avons également mené des recherches dans une autre base de données chinoise (Chongqing VIP Information) jusqu'au 30 avril 2020. Nous avons utilisé des combinaisons de termes relatifs au SRAS, au SRMO, à la COVID-19 et aux médicaments à l'étude, proposées par une bibliothécaire médicale chevronnée (R. C.).

L'inadéquation des données sur l'innocuité suscitant des préoccupations, nous avons aussi mené une recherche élargie dans PubMed, Embase et CENTRAL jusqu'au 19 mars 2020 pour relever des données sur l'innocuité dans des cas d'autres infections respiratoires aiguës. Pour ce faire, nous nous sommes servis de combinaisons de termes ayant trait aux médicaments à l'étude et aux maladies respiratoires infectieuses, ainsi que des termes précis permettant de repérer les ERC. Dans le cadre de nos 2 recherches, nous avons également examiné les références des études publiées admissibles pour y repérer d'autres études pertinentes.

Les critères d'admissibilité des études étaient les suivants : les participants à l'essai devaient avoir reçu un diagnostic de COVID19, de SRAS, de SRMO ou d'une autre maladie respiratoire infectieuse aiguë (annexe 1); l'essai devait comprendre l'administration d'agents antiviraux pertinents pour notre revue (ribavirine, chloroquine, hydroxychloroquine, umifénovir, favipiravir, interféron et lopinavir/ritonavir); il devait évaluer les résultats en lien avec l'efficacité (mortalité, taux de ventilation mécanique, durée de séjour en unité de soins intensifs, durée d'hospitalisation, issues virologiques, et progression de la maladie ou soulagement des symptômes) ou avec l'innocuité (événements symptomatiques ou indésirables graves); et l'étude devait être un ERC, une étude de cohortes ou une étude cas-témoins. Aucune restriction de langue n'a été imposée.

Nous avons retenu les meilleures données probantes provenant des sources suivantes, classées par ordre d'importance : ERC sur la COVID-19; études d'observation sur la COVID-19 comprenant des analyses ajustées; ERC sur le SRAS et le SRMO; études d'observation sur le SRAS et le SRMO comprenant des analyses ajustées; ERC sur les maladies respiratoires infectieuses aiguës autres que le SRAS et le SRMO (pour les résultats d'innocuité seulement) [annexe 1]; études d'observation sans analyses ajustées; et études comparant les agents antiviraux pertinents à d'autres antiviraux. Pour chaque résultat évalué, nous n'avons retenu que les données de meilleure qualité lorsqu'une étude de catégorie supérieure fournissait de meilleures données qu'une étude de catégorie inférieure. En raison des coûts et du temps associés à la transmission des changements de données au groupe de travail pour les lignes directrices, nous n'avons pas tenu compte des données relevées après le 19 avril 2020 si elles étaient de très faible qualité et qu'elles n'étaient pas susceptibles de faire changer les recommandations.

Pour toutes les recherches, 2 personnes ont étudié de manière indépendante les titres et les résumés, puis le texte complet des études prometteuses pour en déterminer l'admissibilité. Tout désaccord a été réglé par la discussion. Deux personnes ont également extrait les données suivantes de façon indépendante : nom des auteurs; année de publication; région ou pays de l'étude; protocole de l'étude; population de patients; taille de chaque groupe; âge et sexe des participants; pourcentage de patients gravement malades; traitement du groupe d'intervention et du groupe témoin; et résultats. Ici aussi, les désaccords ont été réglés par la discussion.

Deux personnes ont évalué de manière indépendante le risque de biais de chaque étude à l'aide d'une version modifiée des critères Cochrane pour les ERC, d'une version modifiée de l'échelle Newcastle-Ottawa pour les études de cohortes et d'un outil créé expressément pour les études cas-témoins ${ }^{10}$. Les évaluateurs ont déterminé si le risque de biais de chaque paramètre était certainement ou probablement faible ou élevé, et ont réglé tout désaccord par la discussion ou, au besoin, par la consultation d'une troisième personne.

L'approche GRADE (Grading of Recommendations, Assessment, Development, and Evaluation) a été utilisée pour déterminer la qualité des données de chaque paramètre à l'étude (tableau 1$)^{13}$. Le taux de mortalité, le taux de ventilation mécanique et la durée de séjour en unité de soins intensifs n'ont été évalués que chez les patients gravement atteints, tandis que la progression de la maladie et les issues en fonction des symptômes n'ont été évaluées que chez les autres patients. En ce qui concerne l'efficacité, nous avons retiré un niveau si les données provenaient d'études sur les patients atteints du SRAS ou du SRMO, en raison de leur caractère indirect. Quant aux résultats sur l'innocuité, nous n'avons pas retiré de niveau pour le caractère indirect des données relatives aux patients atteints 
Tableau 1 : Définition de la qualité des données ${ }^{13}$

\begin{tabular}{ll} 
Qualité & \multicolumn{1}{c}{ Définition } \\
\hline Élevée & $\begin{array}{l}\text { Nous avons pleinement confiance que l'effet réel est } \\
\text { proche de l'estimation. }\end{array}$ \\
\hline Moyenne & $\begin{array}{l}\text { Nous avons une confiance moyenne dans l'estimation } \\
\text { de l'effet, qui est probablement proche du véritable } \\
\text { effet, mais qui pourrait aussi être nettement différente. }\end{array}$ \\
\hline Faible & $\begin{array}{l}\text { Nous avons une confiance limitée dans l'estimation de } \\
\text { l'effet, qui pourrait être nettement différente du } \\
\text { véritable effet. }\end{array}$ \\
\hline Très faible & $\begin{array}{l}\text { Nous avons très peu confiance dans l'estimation de } \\
\text { l'effet, qui est probablement nettement différente du } \\
\text { véritable effet. }\end{array}$
\end{tabular}

du SRAS ou du SRMO, mais nous en avons retiré un pour celui des données relatives aux patients ayant une autre infection respiratoire aiguë. Les données sont présentées dans des tableaux résumant les conclusions selon l'approche GRADE.

\section{Analyse statistique}

Nous avons réalisé notre méta-analyse à l'aide de Review Manager (version 5.3), en nous servant de données relatives à un certain nombre d'événements et de patients tirées d'ERC et d'études de cohortes, ainsi que d'estimations relatives ajustées déclarées dans des études d'observation. Un modèle à effets aléatoires de Mantel-Haenszel nous a permis de calculer le risque relatif (RR) des ERC et des paramètres dichotomiques des études de cohortes. Nous avons également utilisé le modèle à effets aléatoires de DerSimonian et Laird reposant sur la méthode de l'inverse de la variance pour regrouper les RR et les rapports de cotes (RC) ajustés. Nous avons calculé les estimations relatives des études qui avaient publié leurs données initiales au moyen d'une analyse de régression multiple.

Nos populations cibles étaient formées de patients atteints d'une forme grave ou sans signe clinique de gravité de COVID-19. Nous avons utilisé la définition de l'OMS en ce qui a trait à la pneumonie et à la forme grave de COVID-19, soit une fièvre ou une infection respiratoire soupçonnée accompagnée d'au moins un des symptômes suivants : fréquence respiratoire de plus de 30 cycles par minute, détresse respiratoire grave, ou saturation en oxygène périphérique $\left(\mathrm{SpO}_{2}\right) \leq 93 \%$ à l'air ambiant ${ }^{11}$. Pour chaque paramètre, nous avons établi le risque de référence des patients atteints d'une forme grave ou sans signe clinique de gravité de COVID-19 à partir des études publiées, en choisissant les populations les plus représentatives. Nous avons ensuite appliqué des effets relatifs au risque de référence afin d'estimer la différence des risques. Si aucun patient du groupe témoin n'a vécu d'événement indésirable, l'incidence des événements dans le groupe d'intervention et l'intervalle de confiance (IC) de 95\% qui y était associé ont servi à estimer la différence des risques. Lorsque les rapports de risque (HR) étaient fournis, nous avons estimé le risque qu'un événement se produise dans le groupe d'intervention à l'aide du rapport de risque et du risque dans le groupe témoin ${ }^{12}$.

\section{Approbation éthique}

Une approbation éthique n'avait pas à être demandée pour cette revue systématique.

\section{Résultats}

La figure 1 et l'annexe 3 (accessible au www.cmaj.ca/lookup/ doi/10.1503/cmaj.200647/tab-related-content) présentent les diagrammes de l'étude. Nous avons retenu 19 études menées en Chine ( $n=11 ; 7$ étaient des prépublications $\left.{ }^{15,17,18,24,27,28,32}\right)$, en Arabie Saoudite $(n=2)$, à Singapour $(n=1)$, aux États-Unis $(n=1$ [prépublication] $\left.^{19}\right)$, en France $(n=1)$ et au Canada $(n=1)^{14-32}$. Deux des études étaient multirégionales ${ }^{16,29}$. Au total, il y avait 7 ERC, 11 études de cohortes et 1 étude cas-témoins. Les études portaient sur des patients atteints de la COVID-19 $(n=12)$, du SRMO $(n=2)$, du SRAS $(n=4)$ et de l'influenza $(n=1)$. Les traitements administrés étaient les suivants : ribavirine $(n=3)$, hydroxychloroquine $(n=5)$, favipiravir $(n=3)$, interféron $(n=3)$, lopinavir/ritonavir $(n=2)$, umifénovir $(n=1)$, ribavirine et interféron $(n=1)$, et umifénovir et lopinavir/ritonavir $(n=1)$. Le tableau 2 présente les caractéristiques des études retenues. Nous n'avons recensé aucune étude admissible sur l'utilisation de la chloroquine.

Parmi les 7 ERC, 4 ${ }^{15,18,21,26}$ étaient ouverts et ont perdu un niveau parce qu'ils n'ont pas été menés à l'insu (2 de ces études étaient des prépublications ${ }^{15,18}$ ). Un autre ERC n'a pas été mené à l'insu des patients et des médecins (prépublication) ${ }^{17}$. Nous avons déterminé que le risque de biais des 2 derniers $\mathrm{ERC}^{16,28}$ était faible (l'un d'eux était une prépublication ${ }^{28}$ ). La figure 2 présente le risque de biais des ERC, et le tableau 3 et le tableau 4, celui des études d'observation.

Une grande étude épidémiologique chinoise menée auprès de 173 patients atteints d'une forme grave de COVID-19 nous a permis d'estimer que le taux de mortalité de référence était de $10,4 \%^{33}$. Le tableau 5 présente le risque de référence pour chaque paramètre à l'étude.

\section{Ribavirine}

\section{Efficacité}

Deux études de cohortes rétrospectives ${ }^{29,30}$ portant sur 1334 patients atteints du SRAS (gravité variable) ont fourni les estimations pour la mortalité. L'une de ces études a été menée à Hong Kong et au Canada ${ }^{29}$, et l'autre, à Singapour ${ }^{30}$; elles ont été analysées séparément. Le regroupement des résultats indique que la ribavirine exercerait un effet incertain sur la mortalité (RC 0,83, IC de $95 \% 0,49$ à 1,41; annexe 4, figure supplémentaire 1 , accessible au www.cmaj.ca/lookup/doi/10.1503/cmaj.200647/tab -related-content). Une étude cas-témoins menée auprès de 51 patients atteints du SRMO ${ }^{22}$ (gravité variable) a donné des résultats semblables (RC 0,66, IC de $95 \%$ 0,04 à 12,36). Les études sur le SRAS et le SRMO ont toutes fourni des données de très faible qualité quant aux effets du traitement à la ribavirine sur la mortalité chez les patients ayant une forme grave de COVID-19 (annexe 5, tableau supplémentaire 2, accessible au www.cmaj.ca/ lookup/doi/10.1503/cmaj.200647/tab-related-content). 


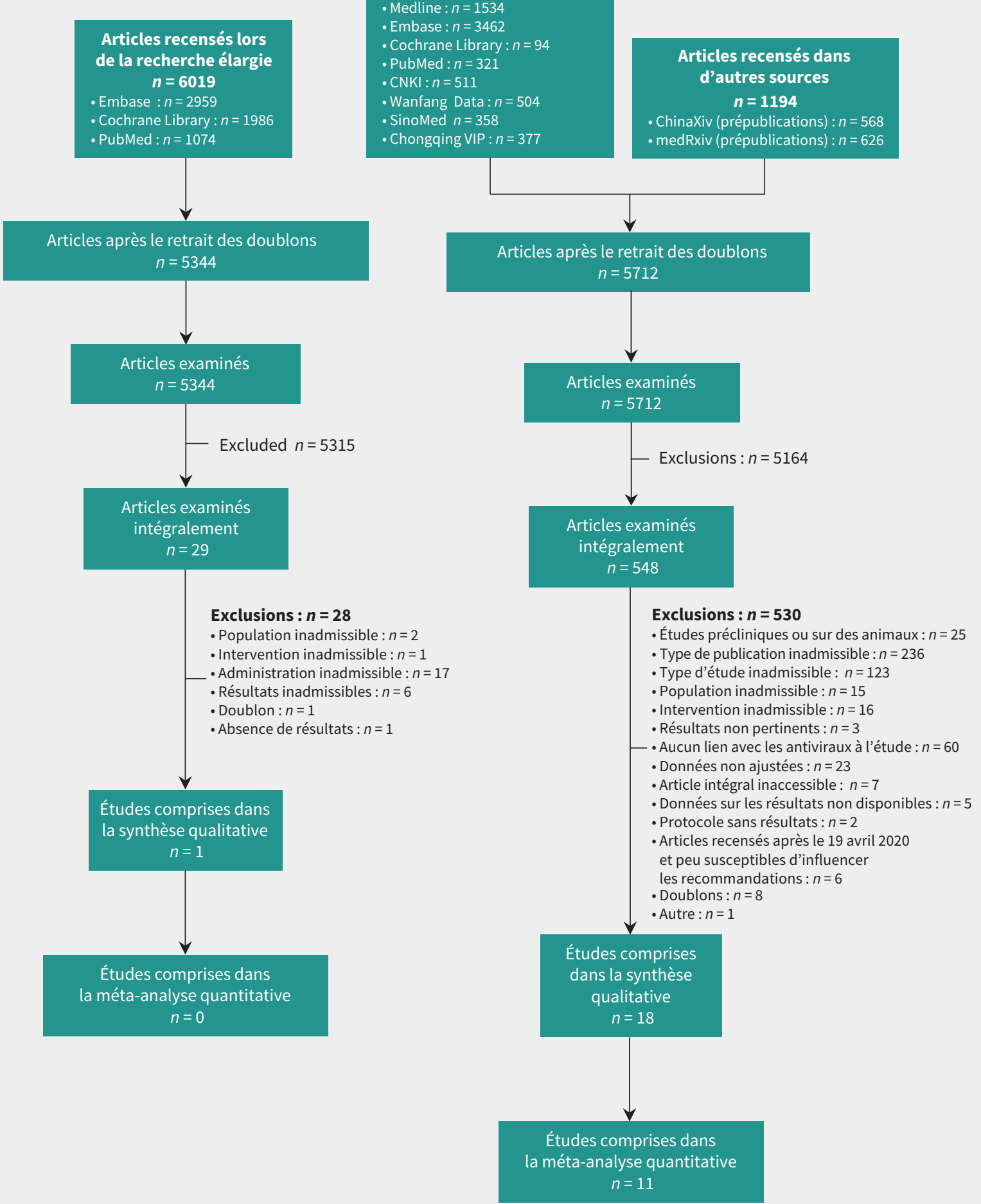

Figure 1 : Diagramme du processus d'inclusion des études. CNKI = China National Knowledge Infrastructure. 


\begin{tabular}{|c|c|c|c|c|c|c|c|c|c|}
\hline Référence & Intervention à l'étude & $\begin{array}{l}\text { Agent antiviral de } \\
\text { comparaison }\end{array}$ & $\begin{array}{l}\text { Modèle } \\
\text { d'étude }\end{array}$ & Pays & $\begin{array}{l}\text { Population } \\
\text { de } \\
\text { participants }\end{array}$ & $\begin{array}{c}\text { Nbre de } \\
\text { participants }\end{array}$ & $\begin{array}{c}\text { Âge, } \\
\text { moyenne } \pm \\
\text { ET }\end{array}$ & $\begin{array}{l}\text { Pourcentage } \\
\text { d'hommes }\end{array}$ & $\begin{array}{l}\text { Pourcentage de } \\
\text { personnes } \\
\text { gravement } \\
\text { atteintes }\end{array}$ \\
\hline \multicolumn{10}{|c|}{ Favipiravir, comparativement au lopinavir/ritonavir } \\
\hline $\begin{array}{l}\text { Cai et al., } \\
2020^{14}\end{array}$ & $\begin{array}{l}\text { Favipiravir } 1600 \mathrm{mg} \text { PO } \\
\text { bid au jour } 1 \text {, puis } \\
600 \mathrm{mg} \text { PO bid aux } \\
\text { jours } 2-14 \text {, plus } \\
\text { interféron- } \alpha \text { ( } 60 \mathrm{\mu g} \text { bid) } \\
\text { par inhalation }\end{array}$ & $\begin{array}{l}\text { Lopinavir/ritonavir } \\
(200 \mathrm{mg} / 50 \mathrm{mg}) 500 \mathrm{mg} \\
\text { PO bid aux jours } 1-14, \\
\text { plus interféron- } 600 \mathrm{\mu g} \\
\text { bid par inhalation }\end{array}$ & Cohortes & Chine & $\begin{array}{l}\text { COVID-19 } \\
\text { sans signe de } \\
\text { gravité }\end{array}$ & 80 & $\begin{array}{c}47,0 \\
(35,8-61,0) \dagger\end{array}$ & 43,8 & 0 \\
\hline \multicolumn{10}{|c|}{ Favipiravir, comparativement à l'umifénovir } \\
\hline $\begin{array}{l}\text { Chen et al., } \\
2020^{15} \ddagger\end{array}$ & $\begin{array}{l}\text { Favipiravir } 1600 \mathrm{mg} \text { PO } \\
\text { bid au jour } 1 \text {, puis } \\
600 \mathrm{mg} \text { PO bid pendant } \\
7-10 \text { jours } \S\end{array}$ & $\begin{array}{l}\text { Umifénovir }(200 \mathrm{mg}) \\
\text { PO tid pendant } \\
7-10 \text { jours }\end{array}$ & ERC & Chine & $\begin{array}{l}\text { COVID-19 de } \\
\text { gravité } \\
\text { variable }\end{array}$ & 236 & N.D. & 46,6 & 11,4 \\
\hline \multicolumn{10}{|c|}{ Favipiravir, comparativement au traitement sans favipiravir } \\
\hline$M D V I^{16}$ & $\begin{array}{l}\text { Favipiravir } 1200 \mathrm{mg} \text { PO } \\
\text { bid au jour } 1, \text { puis } 800 \mathrm{mg} \\
\text { PO bid pendant } 4 \text { jours }\end{array}$ & Placebo & ERC & $\begin{array}{l}\text { Plusieurs } \\
\text { pays }\end{array}$ & $\begin{array}{l}\text { Influenza de } \\
\text { gravité non } \\
\text { précisée }\end{array}$ & 386 & $\begin{array}{c}42,7 \\
(20,0-80,0) ฯ\end{array}$ & 45,3 & N.D. \\
\hline \multicolumn{10}{|c|}{ Hydroxychloroquine, comparativement au traitement sans hydroxychloroquine } \\
\hline $\begin{array}{l}\text { Chen et al., } \\
2020^{17} \ddagger\end{array}$ & $\begin{array}{l}\text { Hydroxychloroquine } \\
(200 \mathrm{mg}) \text { PO bid } \\
\text { pendant } 5 \text { jours }\end{array}$ & $\begin{array}{l}\text { Pas } \\
\text { d'hydroxychloroquine }\end{array}$ & ERC & Chine & $\begin{array}{l}\text { COVID-19 } \\
\text { sans signe de } \\
\text { gravité }\end{array}$ & 62 & $44,7 \pm 15,3$ & 46,8 & 0 \\
\hline $\begin{array}{l}\text { Tang et al., } \\
2020^{18} \ddagger\end{array}$ & $\begin{array}{l}\text { Hydroxychloroquine: } \\
\text { dose initiale de } 1200 \mathrm{mg} \\
\text { par jour pendant } 3 \text { jours, } \\
\text { puis dose d'entretien de } \\
800 \text { mg par jour pour le } \\
\text { reste du traitement } \\
\text { (durée totale du } \\
\text { traitement : } 2 \text { semaines } \\
\text { pour les patients atteints } \\
\text { d'une forme bénigne ou } \\
\text { modérée, ou } 3 \text { semaines } \\
\text { pour les patients atteints } \\
\text { d'une forme grave) }\end{array}$ & $\begin{array}{l}\text { Pas } \\
\text { d'hydroxychloroquine }\end{array}$ & ERC & Chine & $\begin{array}{l}\text { COVID-19 de } \\
\text { gravité } \\
\text { variable }\end{array}$ & 150 & $46,1 \pm 14,7$ & 54,7 & 1,3 \\
\hline $\begin{array}{l}\text { Magagnoli et } \\
\text { al., } 2020^{19} \ddagger\end{array}$ & $\begin{array}{l}\text { Dose } \\
\text { d'hydroxychloroquine } \\
\text { non précisée }\end{array}$ & $\begin{array}{l}\text { Pas } \\
\text { d'hydroxychloroquine }\end{array}$ & Cohortes & É.-U. & $\begin{array}{l}\text { COVID-19 de } \\
\text { gravité } \\
\text { variable }\end{array}$ & 255 & N.D. & 100,0 & N.D. \\
\hline $\begin{array}{l}\text { Mahevas et } \\
\text { al., } 2020^{20} \ddagger\end{array}$ & $\begin{array}{l}\text { Hydroxychloroquine } \\
600 \mathrm{mg} / \text { jour }\end{array}$ & $\begin{array}{l}\text { Pas } \\
\text { d'hydroxychloroquine }\end{array}$ & Cohortes & France & $\begin{array}{l}\text { COVID-19 de } \\
\text { gravité } \\
\text { variable }\end{array}$ & 181 & $\begin{array}{c}60,0 \\
(52,0-68,0) \dagger\end{array}$ & 71,1 & N.D. \\
\hline \multicolumn{10}{|c|}{ Hydroxychloroquine et interféron, comparativement à l'interféron seul } \\
\hline $\begin{array}{l}\text { Chen et al., } \\
2020^{21}\end{array}$ & $\begin{array}{l}\text { Hydroxychloroquine } \\
400 \text { mg PO par jour } \\
\text { pendant } 5 \text { jours, plus } \\
\text { interféron- }- \text { par } \\
\text { inhalation ( } 80,0 \% \text { des } \\
\text { patients ont reçu de } \\
\text { l'umifénovir) }\end{array}$ & $\begin{array}{l}\text { Interféron-a par } \\
\text { inhalation ( } 66,7 \% \text { des } \\
\text { patients ont reçu de } \\
\text { l'umifénovir, et } 13,3 \% \text {, } \\
\text { du lopinavir/ritonavir) }\end{array}$ & ERC & Chine & $\begin{array}{l}\text { COVID-19 } \\
\text { sans signe de } \\
\text { gravité }\end{array}$ & 30 & $48,6 \pm 4,1$ & 70,0 & 0 \\
\hline \multicolumn{10}{|c|}{ Interféron, comparativement au traitement sans interféron; ribavirine, comparativement au traitement sans ribavirine } \\
\hline $\begin{array}{l}\text { Al Ghamdi et } \\
\text { al., } 2016^{22}\end{array}$ & $\begin{array}{l}\text { A : interféron- } \alpha \\
\text { B : interféron- } \beta \\
C \text { : ribavirine }\end{array}$ & $\begin{array}{l}\text { A: pas d'interféron- } \alpha \\
\text { B: pas d'interféron- } \beta \\
\text { C: pas de ribavirine }\end{array}$ & Cas-témoins & $\begin{array}{l}\text { Arabie } \\
\text { saoudite }\end{array}$ & $\begin{array}{l}\text { SRMO de } \\
\text { gravité } \\
\text { variable }\end{array}$ & 51 & $\begin{array}{c}54,0 \\
(36,5-58,0) \dagger\end{array}$ & 78,4 & 37,3 \\
\hline \multicolumn{10}{|c|}{ Interféron et ribavirine, comparativement à la ribavirine seule } \\
\hline $\begin{array}{l}\text { Shalhoub et } \\
\text { al., } 2015^{23}\end{array}$ & $\begin{array}{l}\text { Interféron-a2a } 180 \mu \mathrm{gg} \\
\text { par injection SC une } \\
\text { fois par semaine ou } \\
\text { interféron- } \beta 1 \mathrm{a} 44 \mu \mathrm{g} \\
\text { par injection SC } 3 \text { fois } \\
\text { par semaine, plus } \\
\text { ribavirine PO (dose } \\
\text { initiale de } 2 \mathrm{~g} \text {, puis } \\
600 \mathrm{mg} \mathrm{q} .12 \mathrm{~h} \text { ) }\end{array}$ & $\begin{array}{l}\text { Ribavirine PO (dose } \\
\text { initiale de } 2 \mathrm{~g} \text {, puis } \\
600 \mathrm{mg} \mathrm{q} .12 \mathrm{~h} \text { ) }\end{array}$ & Cohortes & $\begin{array}{l}\text { Arabie } \\
\text { saoudite }\end{array}$ & $\begin{array}{l}\text { SRMO de } \\
\text { gravité } \\
\text { variable }\end{array}$ & 32 & $\begin{array}{c}60,0 \\
(42,0-73,0) \dagger\end{array}$ & 56,0 & N.D. \\
\hline
\end{tabular}


Tableau 2 (partie 2 de 2) : Caractéristiques des études incluses

Dose et administration

Référence

Intervention à l'étude
Agent antiviral de comparaison

Modèle
d'étude

Population

de participants participants

Âge,

Pays oyenne

ET
Pourcentage de personnes gravement atteintes

Interféron et umifénovir, comparativement à l'umifénovir seul

Zhou et al., Interféron- $\alpha 2 \mathrm{~b} 5 \mathrm{mUI}$ Umifénovir $200 \mathrm{mg}$ PO Cohortes Chin $2020^{24} \ddagger$ bid par inhalation, plus tid

COVID-19 de

70

$48,7 \pm 18,1$

N.D. umifénovir 200 mg PO

gravité

tid

variable

Interféron, comparativement au traitement sans interféron

Li et al., Interféron- $\alpha 1 \mathrm{mUI} /$ jour Pas d'interféron

Cohortes Chine

SRAS de

gravité

87

$28,1 \pm 9,5$

18,4

71,3

pendant $6-10$ jours

variable

Lopinavir/ritonavir, comparativement au traitement sans lopinavir/ritonavir

\begin{tabular}{|c|c|c|c|c|c|c|c|c|}
\hline $\begin{array}{l}\text { Cao et al., } \\
2020^{26}\end{array}$ & $\begin{array}{l}\text { Lopinavir/ritonavir } \\
400 \mathrm{mg} / 100 \mathrm{mg} \text { PO bid } \\
\text { pendant } 14 \text { jours }\end{array}$ & $\begin{array}{l}\text { Pas de lopinavir/ } \\
\text { ritonavir }\end{array}$ & ERC & Chine & $\begin{array}{l}\text { COVID-19 de } \\
\text { forme grave }\end{array}$ & 199 & $\begin{array}{c}58,0 \\
(49,0-68,0) \dagger\end{array}$ & 60,3 \\
\hline $\begin{array}{l}\text { Yan et al., } \\
2020^{27} \ddagger\end{array}$ & $\begin{array}{l}\text { Lopinavir/ritonavir } \\
400 \mathrm{mg} / 100 \mathrm{mg} \text { PO bid } \\
\text { pendant } 10 \text { jours ou }\end{array}$ & $\begin{array}{l}\text { Pas de lopinavir/ } \\
\text { ritonavir }\end{array}$ & Cohortes & Chine & $\begin{array}{l}\text { COVID-19 de } \\
\text { gravité } \\
\text { variable }\end{array}$ & 120 & $\begin{array}{c}52,0 \\
(35,0-63,0) \dagger\end{array}$ & 45 \\
\hline
\end{tabular}
plus

gravité

ERC

Li et al.

A : lopinavir/ritonavir Pas de lopinavir/

Chine

(200 mg/50 mg) $500 \mathrm{mg}$ ritonavir ou

PO q. $12 \mathrm{~h}$ pendant d'umifénovir

sans signe de

44

gravité

7-14 jours

B : umifénovir $200 \mathrm{mg}$

PO tid pendant

$7-14$ jours

Ribavirine, comparativement au traitement sans ribavirine

\begin{tabular}{|c|c|c|c|c|c|c|c|c|c|}
\hline $\begin{array}{l}\text { Lau et al., } \\
2009^{29}\end{array}$ & Ribavirine & Pas de ribavirine & Cohortes & $\begin{array}{l}\text { Chine, } \\
\text { Canada }\end{array}$ & $\begin{array}{l}\text { SRAS de } \\
\text { gravité } \\
\text { variable }\end{array}$ & $\begin{array}{l}953 \\
152\end{array}$ & N.D. & 48,$7 ; 36,8$ & N.D. \\
\hline $\begin{array}{l}\text { Leong et al., } \\
2004^{30}\end{array}$ & $\begin{array}{l}\text { Ribavirine } 1,2 \mathrm{~g} \text { PO tid, } \\
\text { ou } 400 \mathrm{mg} \text { par injection } \\
\text { IV q. } 8 \mathrm{~h} \text { pour les } \\
\text { patients gravement } \\
\text { malades ou incapables } \\
\text { de prendre de la } \\
\text { ribavirine par voie } \\
\text { orale; la durée } \\
\text { moyenne du traitement } \\
\text { était de } 5,6 \text { jours }\end{array}$ & Pas de ribavirine & Cohortes & Singapour & $\begin{array}{l}\text { SRAS de } \\
\text { gravité } \\
\text { variable }\end{array}$ & 229 & $39,1 \pm 16,8$ & 31,9 & $\begin{array}{l}20,1 \text { (comme } \\
\text { résultat) }\end{array}$ \\
\hline $\begin{array}{l}\text { Muller et al., } \\
2007^{31}\end{array}$ & $\begin{array}{l}\text { Ribavirine } 2 \mathrm{~g} \text { (dose } \\
\text { initiale) par injection IV, } \\
\text { puis } 1 \mathrm{~g} \mathrm{q.} 8 \mathrm{~h} \text { pendant } \\
4 \text { jours, et ensuite } \\
500 \mathrm{mg} \mathrm{q.} 6 \text { h pendant } \\
3 \text { jours }{ }^{\star \star}\end{array}$ & Pas de ribavirine & Cohortes & Canada & $\begin{array}{l}\text { SRAS de } \\
\text { gravité } \\
\text { variable }\end{array}$ & 306 & N.D. & 37,3 & $41,6 \dagger \dagger$ \\
\hline \multicolumn{10}{|c|}{ Umifénovir, comparativement au traitement sans umifénovir } \\
\hline $\begin{array}{l}\text { Liu et al., } \\
2020^{32} \ddagger\end{array}$ & $\begin{array}{l}\text { Umifénovir (dose non } \\
\text { précisée) }\end{array}$ & Pas d'umifénovir & Cohortes & Chine & $\begin{array}{l}\text { COVID-19 de } \\
\text { gravité } \\
\text { variable }\end{array}$ & 504 & $59,5 \pm 14,9$ & 51,4 & N.D. \\
\hline \multicolumn{10}{|c|}{ 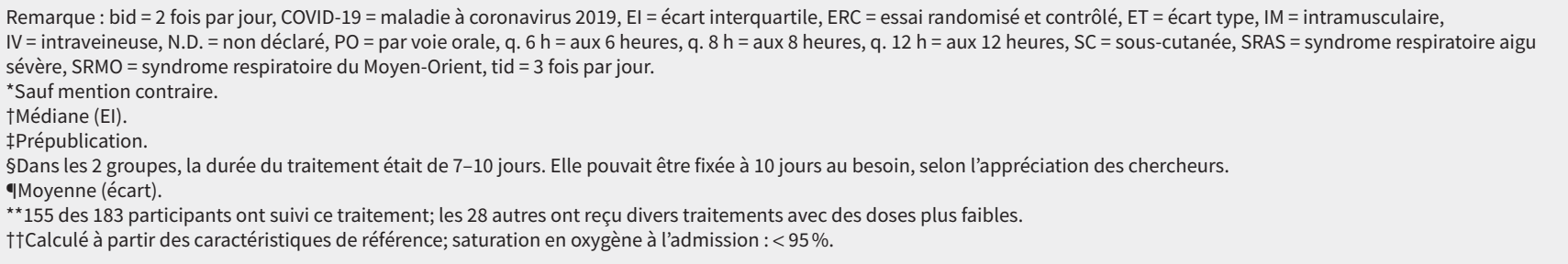 } \\
\hline
\end{tabular}




\section{Innocuité}

Une étude de cohortes rétrospective sur 306 patients ${ }^{31}$ dont la forme de SRAS était de gravité variable a montré que la ribavirine accroît l'incidence de l'anémie (définie comme une diminution du taux d'hémoglobine de $20 \mathrm{~g} / \mathrm{L}$; RC 3,00, IC de $95 \%$ 1,77 à 5,16) et de la bradycardie (définie comme une fréquence cardiaque inférieure à 55 battements par minute; RC 2,30, IC de $95 \% 1,21$ à 4,20). Puisque ces 2 paramètres étaient des marqueurs de substitution (l'anémie pour l'anémie symptomatique, et la bradycardie pour la bradycardie symptomatique), nous avons retiré un niveau en raison du caractère indirect des données et avons jugé leur qualité comme étant très faible (annexe 5 , tableaux supplémentaires 1 et 2 ).

\section{Hydroxychloroquine}

\section{Efficacité}

Trois $\mathrm{ERC}^{17,18,21}$ (dont 2 prépublications ${ }^{17,18}$ ) portant sur 240 patients atteints d'une forme sans signe clinique de gravité de COVID-19 et sur 2 patients atteints d'une forme grave ont comparé le traitement à l'hydroxychloroquine au traitement sans hydroxychloroquine et ont fourni des données de très faible qualité montrant un effet minimal sur la clairance virale au jour 14 (RR 0,98, IC de $95 \%$ 0,89 à 1,07; annexe 4, figure supplémentaire 2), le passage d'une infection bénigne ou modérée à une infection grave ( $R R$ 0,96, IC de $95 \% 0,10$ à 9,66; annexe 4, figure supplémentaire 3) et le rétablissement clinique au jour 7 (RR 1,10, IC de $95 \% 0,44$ à 2,77$)^{17}$. L'hydroxychloroquine pourrait également réduire la durée de la fièvre (différence moyenne [DM] 1 jour de moins, IC de $95 \%$ 0,36 à 1,64 jour de moins; données de très faible qualité; annexe 5 , tableau supplémentaire 3).

Par ailleurs, 2 études d'observation (prépublications) ${ }^{19,20}$ menées auprès de patients atteints de la COVID-19 (181 cas graves et 255 cas de gravité variable) ont fourni des données de très faible qualité quant aux effets de l'hydroxychloroquine sur la mortalité ( $R R 1,48$, IC de $95 \% 0,42$ à 5,24; annexe 4 , figure supplémentaire 4 , et annexe 5 , tableau supplémentaire 4 ). L'une de ces études a également fait état de résultats non concluants sur l'utilisation de l'hydroxychloroquine chez les patients sous ventilation mécanique (annexe 5 , tableau supplémentaire 4$)^{19}$.

\section{Innocuité}

Deux ERC ${ }^{18,21}$ (dont une prépublication ${ }^{18}$ ) portant sur 178 patients atteints d'une forme sans signe clinique de gravité de la COVID-19 et 2 patients atteints d'une forme grave ont indiqué qu'aucun patient du groupe traité sans hydroxychloroquine n'a présenté de diarrhée; cependant, 10,6\% (IC de $95 \%$ 4,0\% à 17,1\%) des patients du groupe traité avec hydroxychloroquine ont eu de la diarrhée (données de faible qualité; annexe 5, tableaux supplémentaires 3 et 4). Un ERC portant sur 62 patients atteints d'une forme sans signe clinique de gravité de la COVID-19 (prépublication) ${ }^{17}$ a signalé la présence de céphalées ou d'éruptions cutanées chez 3,2\% des patients du groupe d'intervention (IC de $95 \% 0 \%$ à 9,4\%), mais chez aucun patient du groupe témoin. Un ERC (prépublication) ${ }^{18}$ mené auprès de 148 patients atteints d'une forme sans signe clinique de gravité de la COVID-19 et de 2 patients atteints d'une forme grave a fait état

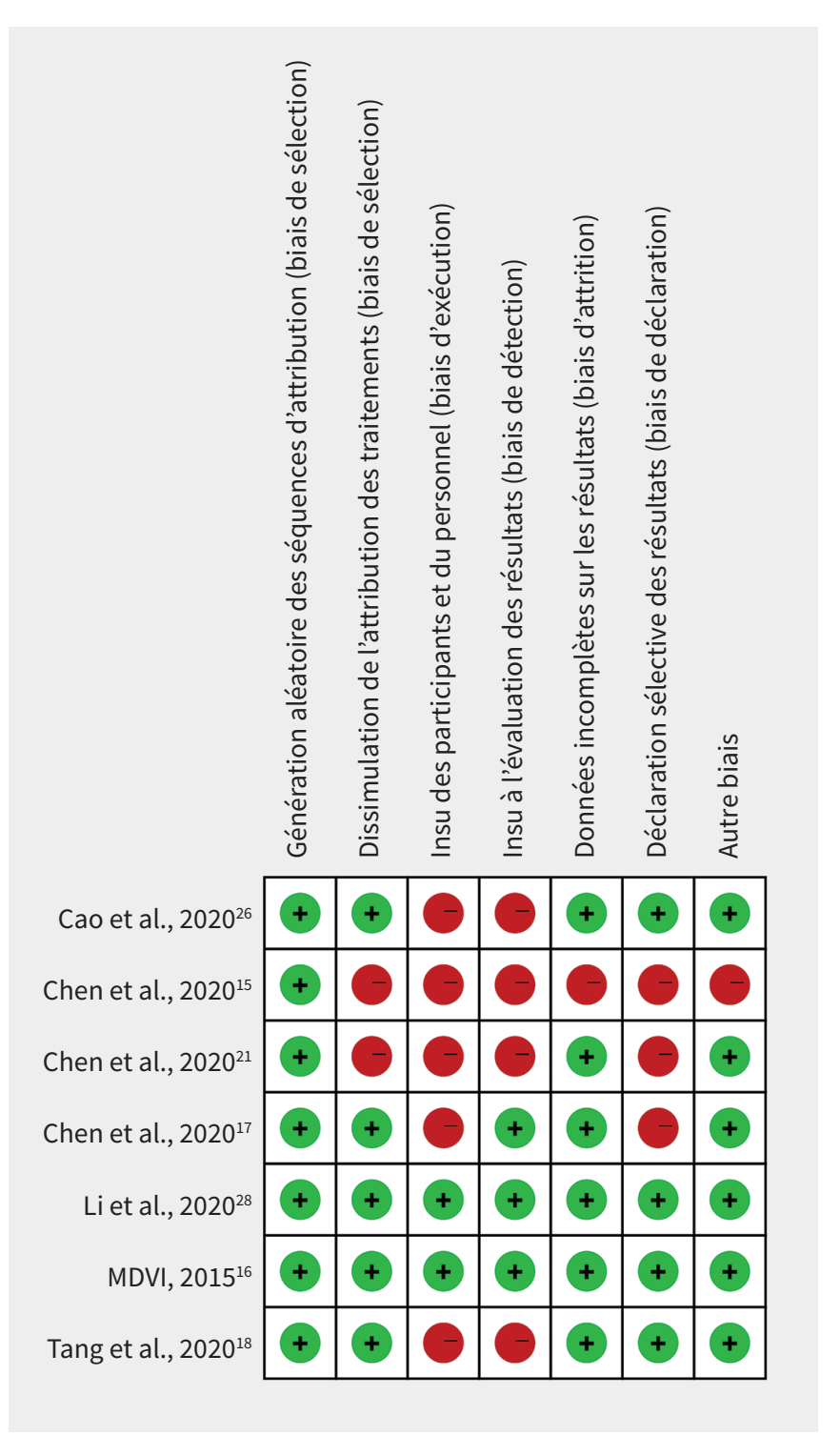

Figure 2 : Évaluation du risque de biais des essais randomisés et contrôlés sélectionnés. Remarque : les références $15,17,18$ et 28 sont des prépublications.

de nausées et de vision trouble ainsi que de vomissements chez $1,4 \%$ (IC de $95 \% 0 \%$ à 4,2\%) et 2,9\% (IC de $95 \% 0 \%$ à $6,8 \%$ ) des patients du groupe d'intervention, respectivement; aucun de ces symptômes n'a été observé au sein du groupe témoin. La qualité des données relatives aux céphalées, aux éruptions cutanées, aux nausées, aux vomissements et à la vision trouble était très faible (annexe 5, tableaux supplémentaires 3 et 4).

\section{Umifénovir}

\section{Efficacité}

Un ERC portant sur 23 patients atteints d'une forme sans signe clinique de gravité de la COVID-19 (prépublication) ${ }^{28}$ a fourni des données limitées montrant que le traitement à l'umifénovir exerçait un effet incertain sur la clairance virale au jour 14, sur la réduction 
Tableau 3 : Évaluation du risque de biais des études de cohortes au moyen d'une version modifiée de l'échelle Newcastle-Ottawa

\begin{tabular}{|c|c|c|c|c|c|c|c|c|}
\hline Étude & $\begin{array}{l}\text { Même } \\
\text { population }\end{array}$ & $\begin{array}{l}\text { Évaluation de } \\
\text { l'exposition }\end{array}$ & $\begin{array}{c}\text { Paramètre } \\
\text { absent au } \\
\text { départ }\end{array}$ & Ajustement & $\begin{array}{c}\text { Évaluation des } \\
\text { facteurs } \\
\text { pronostiques }\end{array}$ & $\begin{array}{l}\text { Évaluation des } \\
\text { résultats }\end{array}$ & Suivi adéquat & $\begin{array}{c}\text { Co- } \\
\text { interventions } \\
\text { semblables }\end{array}$ \\
\hline Cai et al., $2020^{14}$ & Certainement & Probablement & Certainement & Probablement & Probablement & $\begin{array}{c}\text { Probablement } \\
\text { pas }\end{array}$ & Certainement & Probablement \\
\hline Lau et al., $2009^{29}$ & Probablement & Certainement & Certainement & Probablement & Certainement & Certainement & Certainement & Probablement \\
\hline $\begin{array}{l}\text { Leong et al., } \\
2004^{30}\end{array}$ & Certainement & Certainement & Probablement & Probablement & Probablement & Certainement & Certainement & Probablement \\
\hline Li et al., $2005^{25}$ & Probablement & Certainement & Probablement & Probablement & $\begin{array}{c}\text { Probablement } \\
\text { pas }\end{array}$ & Probablement & Certainement & Probablement \\
\hline Muller et al., $2007^{31}$ & Probablement & Certainement & Probablement & Probablement & Certainement & Certainement & Certainement & Probablement \\
\hline $\begin{array}{l}\text { Shalhoub et al., } \\
2015^{23}\end{array}$ & Probablement & Certainement & Certainement & Probablement & $\begin{array}{c}\text { Probablement } \\
\text { pas }\end{array}$ & Probablement & Certainement & Probablement \\
\hline $\begin{array}{l}\text { Zhou et al., } 2020 \\
\text { (prépublication) }^{24}\end{array}$ & Certainement & Certainement & Certainement & Probablement & Probablement & Probablement & Certainement & Probablement \\
\hline $\begin{array}{l}\text { Yan et al., } 2020 \\
\text { (prépublication) }{ }^{27}\end{array}$ & Probablement & Certainement & Probablement & $\begin{array}{c}\text { Probablement } \\
\text { pas }\end{array}$ & Probablement & Probablement & Certainement & $\begin{array}{c}\text { Probablement } \\
\text { pas }\end{array}$ \\
\hline $\begin{array}{l}\text { Liu et al., } 2020 \\
\text { (prépublication) }{ }^{32}\end{array}$ & Certainement & Certainement & Certainement & $\begin{array}{c}\text { Probablement } \\
\text { pas }\end{array}$ & Certainement & Certainement & Certainement & Certainement \\
\hline $\begin{array}{l}\text { Magagnoli et al., } \\
2020 \\
\text { (prépublication) }^{19}\end{array}$ & Certainement & Certainement & Certainement & Probablement & Certainement & Certainement & Certainement & Probablement \\
\hline $\begin{array}{l}\text { Mahevas et al. } \\
2020 \\
\text { (prépublication) }^{20}\end{array}$ & Certainement & Certainement & Certainement & Probablement & Probablement & Probablement & Certainement & Probablement \\
\hline
\end{tabular}

Tableau 4 : Évaluation du risque de biais de l'étude cas-témoins au moyen d'une version modifiée de l'échelle Newcastle-Ottawa

\begin{tabular}{|c|c|c|c|c|c|}
\hline Étude & $\begin{array}{l}\text { Évaluation de } \\
\text { l'exposition }\end{array}$ & $\begin{array}{l}\text { Paramètre présent } \\
\text { chez les cas, mais } \\
\text { pas chez les } \\
\text { témoins }\end{array}$ & Sélection des cas & $\begin{array}{l}\text { Sélection des } \\
\text { témoins }\end{array}$ & $\begin{array}{l}\text { Appariement ou } \\
\text { ajustement }\end{array}$ \\
\hline Al Ghamdi et al., $2016^{22}$ & Certainement & Certainement & Certainement & Certainement & Probablement \\
\hline
\end{tabular}

de la toux et de la fièvre au jour 7 , ainsi que sur la progression vers une forme grave de l'infection (annexe 5, tableau supplémentaire 5). Il a également fourni des données encore plus faibles et plus indirectes montrant un retard de la clairance virale chez les patients atteints d'une forme grave de la COVID-19 (annexe 5, tableau supplémentaire 6). Une étude d'observation réalisée à Wuhan (Chine) et portant sur 504 patients dont la forme de COVID-19 était de gravité variable (prépublication) ${ }^{32}$ a mis en évidence une diminution importante de la mortalité chez ceux qui avaient reçu de l'umifénovir (RC 0,18, IC de $95 \%$ 0,08 à 0,45). Nous avons toutefois déterminé que cette étude d'observation fournissait des données de très faible qualité en raison de sa conception et de la prise en considération sous-optimale de la gravité de l'infection (annexe 5, tableau supplémentaire 6).

\section{Innocuité}

L'ERC mené auprès de 23 patients atteints d'une forme sans signe clinique de gravité de COVID-19 (prépublication) ${ }^{28}$ a indiqué qu'aucun patient du groupe d'intervention et du groupe témoin n'a présenté de diarrhée ou une perte d'appétit (données de très faible qualité; annexe 5, tableaux supplémentaires 5 et 6).

\section{Favipiravir}

\section{Efficacité}

Un ERC portant sur 236 patients (prépublication) ${ }^{15}$ dont la forme de COVID-19 était de gravité variable (88,6\% avaient une infection sans signe de gravité) a comparé le traitement au favipiravir à celui à l'umifénovir, et a indiqué que le favipiravir pourrait faire augmenter le rétablissement clinique au jour 7 (RR 1,18, IC de 95\% 0,95 à 1,48; données de très faible qualité; annexe 5 , tableau supplémentaire 7 ). Une étude d'intervention non randomisée menée auprès de 80 patients présentant une forme sans signe clinique de gravité de COVID-1914 a comparé le traitement au favipiravir à celui au lopanivir/ritonavir et a montré que le favipiravir fait augmenter la clairance virale au jour 7 (rapport de risque 3,43, IC de 95\% 1,16 à 10,15; données de très faible qualité; annexe 5, tableaux supplémentaires 7 et 8 ). 
Tableau 5 : Risque de référence pour les patients atteints d'une forme grave ou sans signe clinique de gravité de COVID-19

\begin{tabular}{|c|c|c|}
\hline Paramètre & $\begin{array}{l}\text { Patients atteints } \\
\text { d'une forme sans } \\
\text { signe clinique de } \\
\text { gravité de } \\
\text { COVID-19 }\end{array}$ & $\begin{array}{l}\text { Patients atteints } \\
\text { d'une forme } \\
\text { grave de } \\
\text { COVID-19 }\end{array}$ \\
\hline Mortalité & S.O. & $10,4 \% 33$ \\
\hline Durée d'hospitalisation & 11 jours $^{33}$ & 13 jours $^{33}$ \\
\hline $\begin{array}{l}\text { Durée de séjour en unité } \\
\text { de soins intensifs }\end{array}$ & S.O. & 11 jours $^{26}$ \\
\hline Ventilation mécanique & S.O. & $38,7 \%^{33}$ \\
\hline $\begin{array}{l}\text { Clairance virale au } \\
\text { jour } 14\end{array}$ & $\begin{array}{c}71,4 \% \\
\text { (prépublication) }^{28}\end{array}$ & $56,3 \% 26$ \\
\hline Clairance virale au jour 7 & $\begin{array}{c}71,4 \% \\
\text { (prépublication) }^{28}\end{array}$ & $32,4 \% \%^{\star 26}$ \\
\hline $\begin{array}{l}\text { Passage d'une forme } \\
\text { sans signe clinique de } \\
\text { gravité à une forme grave }\end{array}$ & $\begin{array}{c}14,3 \% \\
\text { (prépublication) }^{28}\end{array}$ & S.O. \\
\hline
\end{tabular}

\section{Innocuité}

Un ERC portant sur 386 patients atteints de l'influenza ${ }^{16}$ a fourni des données ne permettant pas de conclure si le favipiravir causait de la diarrhée (différence de risques [DR] 3 de moins par 1000 personnes, IC de $95 \% 31$ de moins à 64 de plus par 1000 personnes; données de faible qualité; annexe 5 , tableaux supplémentaires 9 et 10 ).

\section{Interféron- $\alpha$ et interféron- $\beta$}

\section{Efficacité}

Une étude de cohortes rétrospective sur 70 patients dont la forme de COVID-19 était de gravité variable (prépublication) ${ }^{24}$ a comparé l'administration combinée d'interféron- $\alpha$ et d'umifénovir au traitement à l'umifénovir seul et a fourni des données sur la clairance virale et la durée d'hospitalisation. Nous avons réalisé une analyse de régression linéaire multiple fondée sur les données initiales (annexe 6, accessible au www.cmaj.ca/lookup/doi/10.1503/ cmaj.200647/tab-related-content). Nous avons constaté que les patients ayant reçu de l'interféron- $\alpha$ avaient tendance à présenter une clairance virale plus rapide que les autres (DM 4,6 jours, IC de $95 \%-0,5$ à 9,6 jours) et une durée d'hospitalisation plus courte (DM 4,4 jours, IC de $95 \%-1,5$ à 10,3 jours; annexe 5, tableaux supplémentaires 11 et 12). Cependant, les données étaient de très faible qualité en raison de l'utilisation concomitante d'umifénovir et de l'imprécision de résultats.

Une étude de cohortes rétrospective ${ }^{23}$ et une étude castémoins ${ }^{22}$ menées auprès d'un total de 83 patients atteints de SRMO dont la forme était de gravité variable ont fourni des données de très faible qualité laissant penser que la mortalité pourrait être réduite par l'interféron- $\alpha$ (RC 0,23, IC de $95 \% 0,04$ à
1,32; annexe 4, figure supplémentaire 5 , et annexe 5 , tableau supplémentaire 14) et l'interféron- $\beta$ ( RC 0,37, IC de $95 \% 0,07$ à 2,05 ; annexe 4 , figure supplémentaire 6 ; et annexe 5 , tableau supplémentaire 15).

\section{Innocuité}

Une étude de cohortes rétrospective portant sur 87 patients atteints du SRAS ${ }^{25}$ a fourni des données de très faible qualité quant à l'effet de l'interféron- $\alpha$ sur le besoin de facteur de croissance granulocytaire chez les patients présentant une leucopénie (annexe 5, tableaux supplémentaires 13 et 14). Nous n'avons trouvé aucune donnée sur l'innocuité de l'interféron- $\beta$.

\section{Lopinavir/ritonavir}

\section{Efficacité}

Un ERC mené auprès de 199 patients ayant une forme grave de COVID-19 (prépublication) ${ }^{26}$ a comparé le traitement au lopinavir/ritonavir au traitement sans lopinavir/ritonavir et s'est intéressé à la mortalité, à la clairance virale au jour 14, à la ventilation mécanique, à la durée de séjour en unité de soins intensifs et à la durée d'hospitalisation. Un autre ERC a comparé ces 2 traitements chez 28 patients ayant une forme sans signe clinique de gravité de COVID-19 (prépublication) ${ }^{28}$ et s'est penché sur la mortalité, la clairance virale au jour 14, le soulagement de la toux au jour 7 , le passage d'une infection bénigne ou modérée à une infection grave, et la fièvre au jour 7. Comme il n'y a eu aucun décès dans le deuxième ERC, nous n'avons tenu compte que des données sur la mortalité du premier ERC, qui portait sur les patients gravement malades. Une autre étude d'observation portant sur 120 patients dont la forme de COVID-19 était de gravité variable a étudié la clairance virale au jour 23 (prépublication ${ }^{27}$. Nous avons réalisé une méta-analyse sur la clairance virale au jour 14 (annexe 4, figure supplémentaire 7).

Chez les patients ayant une forme sans signe clinique de gravité de COVID-19, le lopinavir/ritonavir ne réduirait que peu ou pas du tout la clairance virale au jour 14 (DR $-0,7 \%$, IC de $95 \%-17,1 \%$ à $20,7 \%$; données de faible qualité ${ }^{26}$ $\left[\right.$ prépublication] ${ }^{28}$ ). L'étude d'observation laisse penser qu'il est possible que la clairance virale soit accrue au jour 23 avec le traitement au lopinavir/ritonavir, mais les données n'ont pas été ajustées selon la gravité de l'infection, ce qui fait que cette conclusion est de très faible qualité (prépublication) ${ }^{27}$. Nous avons également obtenu des données de très faible qualité montrant que le lopinavir/ritonavir exerçait un effet incertain sur le soulagement de la toux au jour 7 , le passage à une infection grave, la fièvre au jour 7 et la durée d'hospitalisation ${ }^{14}$ (prépublication) ${ }^{19}$ (annexe 5, tableau supplémentaire 16).

Chez les patients gravement atteints par la COVID-19, le lopinavir/ritonavir pourrait réduire légèrement la mortalité (DR 2,4\% moins de décès, IC de $95 \%$ diminution de $5,7 \%$ à augmentation de 3,1\%; données de faible qualité) et diminuer la durée de séjour en unité de soins intensifs (DR 5 jours de moins, IC de $95 \% 0$ à 9 jours; données de faible qualité) et la durée d'hospitalisation (DR 1 jour de moins, IC de $95 \% 0$ à 2 jours; données de faible qualité ${ }^{14}$; annexe 5 , tableau supplémentaire 17 ). 


\section{Innocuité}

Un ERC portant sur 194 patients ayant une forme grave de COVID-1926 et un autre ERC portant sur 28 patients ayant une forme sans signe clinique de gravité de COVID-19 (prépublication) ${ }^{28}$ n'ont signalé aucun cas de diarrhée chez les témoins. L'incidence de la diarrhée chez les patients du groupe d'intervention était de $6,0 \%$ (IC de $95 \% 1,7 \%$ à $10,4 \%{ }^{26}$ [prépublication] ${ }^{28}$; données de qualité moyenne; annexe 5 , tableaux supplémentaires 16 et 17). L'ERC de 194 patients $^{26}$ a montré que le lopinavir/ritonavir augmentait probablement les nausées (DM 9,5\%, IC de $95 \% 3,6 \%$ à 15,4\%) et les vomissements (DM 6,3\%, IC de 95\% 1,4\% à 11,2\% [données de qualité moyenne dans les 2 cas]; annexe 5, tableaux supplémentaires 16 et 17). Cette étude a également fourni des données de très faible qualité soulevant la possibilité d'une augmentation des maux d'estomac (annexe 5, tableaux supplémentaires 16 et 17$)$.

\section{Interprétation}

Notre revue systématique ne nous a pas permis de trouver de données concluantes sur les bénéfices associés aux agents antiviraux chez les patients atteints de la COVID-19. Nous n'avons obtenu aucune donnée directe sur le traitement à la ribavirine en cas de COVID-19, et les résultats des études sur des personnes atteintes du SRAS ou du SRMO n'appuient pas l'idée selon laquelle le traitement à la ribavirine réduit la mortalitée $22,29,30$. Nous avons aussi constaté que l'interféron n'a pas de bénéfices sur la clairance virale ou la durée d'hospitalisation en cas de COVID-19.

Chez les patients ayant une forme sans signe clinique de gravité de COVID-19, l'hydroxychloroquine et l'umifénovir n'ont pas été associés à des bénéfices en ce qui concerne la clairance virale, la progression de la maladie et le soulagement des symptômes. Chez les patients atteints d'une forme grave, l'hydroxychloroquine n'a pas réduit la mortalité ni le recours à la ventilation mécanique. Selon une étude d'observation aux données de très faible qualité, l'umifénovir réduirait la mortalité. Des données de très faible qualité ont laissé entendre que le favipiravir pourrait accélérer le rétablissement clinique, comparativement à l'umifénovir, et accélérer la clairance virale, comparativement au lopinavir/ritonavir.

Les ERC retenus se sont intéressés à l'administration de lopinavir/ritonavir chez les patients ayant une forme grave ou sans signe clinique de gravité de COVID-19. Toutefois, l'échantillon de l'ERC sur les patients non gravement atteints ne comptait que 28 personnes, ce qui a donné de grands intervalles de confiance pour tous les paramètres à l'étude. Selon l'ERC sur les patients gravement atteints, il est possible que le lopinavir/ritonavir réduise la mortalité au jour 28 , la durée de séjour en unité de soins intensifs et la durée d'hospitalisation, mais les données étaient de faible qualité. Des données de qualité moyenne ont cependant montré une augmentation considérable des effets gastrointestinaux indésirables avec le lopinavir/ritonavir.

Les effets indésirables associés à chacun de ces médicaments demeurent préoccupants. Les principales sources de préoccupation en lien avec le lopinavir/ritonavir sont les troubles gastro- intestinaux et les interactions médicamenteuses potentielles. L'hydroxychloroquine et la chloroquine allongent quant à elles l'intervalle QT, et selon des études de cas tirées de la base de données FDA Adverse Event Reporting System, la Food and Drug Administration des États-Unis a lancé une mise en garde contre le risque de décès cardiocirculatoire soudain associé à la prise de ces médicaments, avec ou sans azithromycine, en cas de COVID-1934.

Les forces de notre revue résident dans notre équipe formée de personnes ayant une grande expertise méthodologique, pharmacologique et clinique développée dans le cadre du traitement direct de patients atteints de la COVID-19, la prise en considération du caractère direct et indirect des données, la recherche documentaire exhaustive et à jour, l'évaluation de l'admissibilité et du risque de biais des études, et l'abstraction des données provenant de doublons. Grâce à l'approche GRADE, nous avons ciblé les meilleures données disponibles et avons prêté une attention particulière au caractère direct ou indirect de celles-ci. Cette approche nous a également amenés à nous concentrer sur les effets absolus et à présenter les données de façon succincte et informative dans des tableaux.

Depuis le début de la pandémie de COVID-19, des efforts ont été déployés pour résumer les données disponibles sur les traitements antiviraux. Bien que des revues des études sur le traitement du SRMO et du SRAS avec des agents antiviraux aient été publiées, elles n'ont pas été regroupées ou appliquées au contexte de la COVID-19 au moyen d'une méthodologie rigoureuse ${ }^{35-41}$. Par exemple, l'Agence de la santé publique du Canada (ASPC) a publié un examen rapide sur l'efficacité et l'innocuité des antiviraux et des anticorps dans la lutte contre le coronavirus $^{35}$. Les auteurs se sont intéressés à tous les anticorps et antiviraux connus pour trouver un traitement potentiel et ont recensé des études de toutes sortes, dont des études précliniques (sur des animaux). Ce type d'efforts, y compris ceux de l'ASPC, est limité à 2 égards. D'abord, les chercheurs n'ont pas utilisé un système officiel, comme l'approche GRADE, pour évaluer la certitude des données. Pour que les données d'un groupe de patients puissent être appliquées à un autre groupe, le système utilisé doit suivre une méthode officielle pour l'évaluation du caractère indirect des données - cela permet de savoir à quel point la prudence est de mise lorsque vient le temps d'appliquer des données tirées, par exemple, d'études sur le SRAS et le SRMO à la COVID-19. Cette évaluation est cruciale pour les décideurs, qui savent alors à quel point les données dont ils disposent sont crédibles. Ensuite, les chercheurs n'ont pas calculé le risque absolu de ces médicaments en fonction du risque de référence des patients atteints de la COVID-19, ce qui a limité leur application à la prise en charge concrète de la COVID19. Notre revue a tenu compte de ces enjeux.

Les résultats des ERC en cours (prépublication) ${ }^{42}$ feront certainement augmenter la qualité des données et pourraient fournir des données concluantes sur les bénéfices associés à ces médicaments, ce que notre étude n'a pas été en mesure de faire. Toutefois, les cliniciens ont besoin de directives dès maintenant, et c'est ce que leur donne notre étude.

Des études in vitro et des études sur les animaux portant sur des traitements antiviraux contre la COVID-19 ont montré que le 
remdésivir, la chloroquine et l'hydroxychloroquine exerçaient des effets inhibiteurs sur le SRAS-CoV-2, ce qui est prometteur ${ }^{43-45}$. Des chercheurs ont également signalé que l'umifénovir empêche la reproduction du SRAS-CoV-2 dans des cultures cellulaires ${ }^{46}$. Des études sur des cultures cellulaires et des animaux ont mis en évidence l'action de la ribavirine, d'une dose élevée d'interféron ${ }^{47,48}$ et du lopinavir/ritonavir ${ }^{49-51}$ contre les coronavirus. Or, ces études ne suffisent pas pour justifier l'utilisation de ces médicaments chez des humains atteints de la COVID-19 compte tenu des effets indésirables qu'ils peuvent avoir ${ }^{52}$.

\section{Limites de l'étude}

La principale limite de notre revue réside dans la très faible qualité des données actuellement disponibles sur les bénéfices et les préjudices associés aux agents antiviraux, ce qui laisse place à une certaine incertitude. Cette dernière découle surtout des estimations des effets relatifs, mais également des estimations du risque de référence pour les patients atteints de COVID-19, qui viennent d'études individuelles menées sur des échantillons de petite taille.

De plus, nous avons restreint notre revue aux interventions pour lesquelles des données ont été publiées. Cependant, en raison des doutes entourant certains agents antiviraux, nos conclusions quant à l'incertitude de leurs bénéfices peuvent également s'appliquer aux combinaisons de médicaments, qui, par ailleurs, sont plus susceptibles que les antiviraux seuls d'occasionner des préjudices.

\section{Conclusion}

Cette revue fournit des données pour appuyer la rédaction de lignes directrices sur la COVID-19. À l'heure actuelle, rien ne prouve de façon convaincante que les traitements antiviraux apportent des bénéfices importants, bien que les données propres à chaque traitement n'excluent pas cette possibilité. Étant donné le très faible risque de décès des patients ayant une forme sans signe clinique de gravité de COVID-19, les agents antiviraux ne réduisent pas de façon considérable la mortalité chez cette population. Pour pouvoir administrer avec confiance des traitements antiviraux, il faudra réaliser d'autres ERC faisant état de bénéfices pour les patients.

\section{Références}

1. Novel coronavirus (2019-nCoV) situation report - 22. Genève : Organisation mondiale de la Santé; 2020. Accessible ici : www.who.int/docs/default-source/ coronaviruse/situation-reports/20200211-sitrep-22-ncov.pdf?sfvrsn=fb6d49b1_2 (consulté le 3 mars 2020).

2. Coronavirus disease 2019 (COVID-19) situation report - 101. Genève : Organisation mondiale de la Santé; 2020. Accessible ici : www.who.int/docs/default -source/coronaviruse/situation-reports/20200430-sitrep-101-covid-19.pdf?sfursn =2ba4e093_2 (consulté le $1^{\mathrm{er}}$ mai 2020).

3. Coronaviridae Study Group of the International Committee on Taxonomy of Viruses. The species Severe acute respiratory syndrome-related coronavirus: classifying 2019-nCoV and naming it SARS-CoV-2. Nat Microbiol 2020;5:536-544.

4. Zhu N, Zhang D, Wang W, et al.; China Novel Coronavirus Investigating and Research Team. A novel coronavirus from patients with pneumonia in China, 2019. N Engl J Med 2020;382:727-33.

5. Lu R, Zhao X, Li J, et al. Genomic characterisation and epidemiology of 2019 novel coronavirus: implications for virus origins and receptor binding. Lancet 2020;395:565-74.
6. Initial public health response and interim clinical guidance for the 2019 novel coronavirus outbreak - United States, December 31, 2019-February 4, 2020. [Erratum publié dans MMWR Morb Mortal Wkly Rep 2020;69:173]; révision le 13 février 2020. MMWR Morb Mortal Wkly Rep 2020;69:140-6.

7. Prise en charge clinique de la COVID-19 chez l'humain (conseils pour les essais cliniques, version 7), $\mathrm{n}^{\circ} 184$. Beijing (Chine) : Commission nationale de la santé de la République populaire de Chine; 2020. Accessible ici : www.nhc.gov.cn/xcs /zhengcwj/202003/46c9294a7dfe4cef80dc7f5912eb1989/files/ce3e6945832a438 eaae415350a8ce964.pdf (consulté le 4 mars 2020).

8. Ye Z, Rochwerg B, Wang Y, et al. Traitement des patients atteints d'une forme modérée ou grave de maladie à coronavirus 2019 : Ligne directrice fondée sur des données probantes. CMAJ 29 avril 2020. [Cyberpublication avant impression]. doi : $10.1503 / \mathrm{cmaj} .200648$.

9. Wang Y, Zhang D, Du G, et al. Remdesivir in adults with severe COVID-19: a randomised, double-blind, placebo-controlled, multicentre trial. Lancet 29 avril 2020. doi : 10.1016/S0140-6736(20)31022-9.

10. Methodological resources. Ottawa : Evidence Partners. Accessible ici : www. evidencepartners.com/resources/methodological-resources/ (consulté le 28 mars 2020).

11. Clinical management of COVID-19: interim guidance. Genève : Organisation mondiale de la Santé; 2020. Accessible ici : www.who.int/publications/i/item/ clinical-management-of-covid-19 (consulté le 17 mars 2020).

12. Skoetz N, Goldkuhle M, van Dalen EC, et al.; GRADE Working Group. GRADE guidelines 27: how to calculate absolute effects for time-to-event outcomes in summary of findings tables and Evidence Profiles. J Clin Epidemiol 2020; 118:124-31.

13. Guyatt GH, Oxman AD, Vist GE, et al.; GRADE Working Group. GRADE: an emerging consensus on rating quality of evidence and strength of recommendations. $B M J$ 2008;336:924-6.

14. Cai Q, Yang M, Liu D, et al. Experimental treatment with favipiravir for COVID-19: an open-label control study. Engineering (Beijing) 18 mars 2020. [Cyberpublication avant impression]. doi : 10.1016/j.eng.2020.03.007.

15. Chen C, Zhang Y, Huang J, et al. Favipiravir versus arbidol for COVID-19: a randomized clinical trial [prépublication]. medRxiv 15 avril 2020. doi : 10.1101 /2020.03.17.20037432.

16. MDVI. Dose-finding study of favipiravir in the treatment of uncomplicated influenza. ClinicalTrials.gov : NCT01068912. 2014, mise à jour le 18 novembre 2015. Accessible ici : clinicaltrials.gov/show/NCT01068912 (consulté le 28 mars 2020).

17. Chen Z, Hu J, Zhang Z, et al. Efficacy of hydroxychloroquine in patients with COVID-19: results of a randomized clinical trial [prépublication]. medRxiv 10 avril 2020. doi : 10.1101/2020.03.22.20040758.

18. Tang W, Cao Z, Han M, et al. Hydroxychloroquine in patients with COVID-19: an open-label, randomized, controlled trial [prépublication]. medRxiv 7 mai 2020. doi : 10.1101/2020.04.10.20060558.

19. Magagnoli J, Narendran S, Pereira F, et al. Outcomes of hydroxychloroquine usage in United States veterans hospitalized with COVID-19 [prépublication]. medRxiv 23 avril 2020. doi : 10.1101/2020.04.16.20065920.

20. Mahevas M, Tran V-T, Roumier M, et al. No evidence of clinical efficacy of hydroxychloroquine in patients hospitalized for COVID-19 infection with oxygen requirement: results of a study using routinely collected data to emulate a target trial [prépublication]. medRxiv 14 avril 2020. doi : 10.1101/2020.04.10.20060699.

21. Chen J, Liu D, Liu L, et al. A pilot study of hydroxychloroquine in treatment of patients with moderate COVID-19. J Zhejiang Univ 2020;49. doi : 10.3785/j. issn.1008-9292.2020.03.03.

22. Al Ghamdi M, Alghamdi KM, Ghandoora Y, et al. Treatment outcomes for patients with Middle Eastern Respiratory Syndrome Coronavirus (MERS CoV) infection at a coronavirus referral center in the Kingdom of Saudi Arabia. BMC Infect Dis 2016;16:174.

23. Shalhoub S, Farahat F, Al-Jiffri A, et al. IFN- $\alpha 2$ a or IFN- $\beta 1$ 1a in combination with ribavirin to treat Middle East respiratory syndrome coronavirus pneumonia: a retrospective study. J Antimicrob Chemother 2015;70:2129-32.

24. Zhou Q, Wei X-S, Xiang X, et al. Interferon- $\alpha 2 b$ treatment for COVID-19 [prépublication]. medRxiv 10 avril 2020. doi : 10.1101/2020.04.06.20042580.

25. Li J, Wu H, Huang Z, et al. Clinical study of interferon- $\alpha$ in the treatment of severe acute respiratory syndrome. Clinical Medicine of China 2005;21:109-10.

26. Cao B, Wang Y, Wen D, et al. A trial of lopinavir-ritonavir in adults hospitalized with severe COVID-19. N Engl J Med 2020;382:1787-99.

27. Yan D, Liu X-Y, Zhu Y-N, et al. Factors associated with prolonged viral shedding and impact of lopinavir/ritonavir treatment in patients with SARS-CoV-2 infection [prépublication]. medRxiv 30 mars 2020. doi : 10.1101/2020.03.22.20040832. 
28. Li Y, Xie Z, Lin W, et al. An exploratory randomized, controlled study on the efficacy and safety of lopinavir/ritonavir or arbidol treating adult patients hospitalized with mild/moderate COVID-19 (ELACOI) [prépublication]. medRxiv 15 avril 2020. doi : 10.1101/2020.03.19.20038984.

29. Lau EH, Cowling BJ, Muller MP, et al. Effectiveness of ribavirin and corticosteroids for severe acute respiratory syndrome. Am J Med 2009;122:1150.e11-1150.e21.

30. Leong HN, Ang B, Earnest A, et al. Investigational use of ribavirin in the treatment of severe acute respiratory syndrome, Singapour, 2003. Trop Med Int Health 2004;9:923-7.

31. Muller MP, Dresser L, Raboud J, et al.; Canadian SARS Research Network. Adverse events associated with high-dose ribavirin: evidence from the Toronto outbreak of severe acute respiratory syndrome. Pharmacotherapy 2007;27:494-503.

32. Liu Q, Fang $X$, Tian L, et al. The effect of arbidol hydrochloride on reducing mortality of COVID-19 patients: a retrospective study of real world data from three hospitals in Wuhan [prépublication]. medRxiv 17 avril 2020. doi : 10.1101 /2020.04.11.20056523.

33. Guan W-J, Ni Z-Y, Hu Y, et al. Clinical characteristics of coronavirus disease 2019 in China. N Engl J Med 2020;382:1708-20.

34. FDA Drug Safety Communication. FDA cautions against use of hydroxychloroquine or chloroquine for COVID-19 outside of the hospital setting or a clinical trial due to risk of heart rhythm problems - Does not affect FDA-approved uses for malaria, lupus, and rheumatoid arthritis [annonce concernant la sûreté]. Rockville (Maryland) : Food and Drug Administration des États-Unis; 24 avril 2020. Accessible ici : www.fda.gov/media/137250/download (consulté le 1er mai 2020).

35. Rios P, Radhakrishnan A, Antony J, et al. Effectiveness and safety of antiviral or antibody treatments for coronavirus: a rapid review [prépublication]. medRxiv 25 mars 2020. doi : 10.1101/2020.03.19.20039008.

36. Gross AE, Bryson ML. Oral ribavirin for the treatment of noninfluenza respiratory viral infections: a systematic review. Ann Pharmacother 2015;49:1125-35.

37. Momattin H, Al-Ali AY, Al-Tawfiq JA. A systematic review of therapeutic agents for the treatment of the Middle East respiratory syndrome coronavirus (MERS-CoV). Travel Med Infect Dis 2019;30:9-18.

38. Morra ME, Van Thanh L, Kamel MG, et al. Clinical outcomes of current medical approaches for Middle East respiratory syndrome: a systematic review and meta-analysis. Rev Med Virol 2018;28:e1977.

39. Soares-Weiser K, Thomas S, Thomson G, et al. Ribavirin for Crimean-Congo hemorrhagic fever: systematic review and meta-analysis. BMC Infect Dis 2010;10:207.
40. Stockman LJ, Bellamy R, Garner P. SARS: systematic review of treatment effects. PLoS Med 2006;3:e343.

41. Momattin H, Mohammed K, Zumla A, et al. Therapeutic options for Middle East respiratory syndrome coronavirus (MERS-CoV) - possible lessons from a systematic review of SARS-CoV therapy. Int J Infect Dis 2013;17:e792-8.

42. Belhadi D, Peiffer-Smadja N, Lescure F-X, et al. A brief review of antiviral drugs evaluated in registered clinical trials for COVID-19 [prépublication]. medRxiv 27 mars 2020. doi : 10.1101/2020.03.18.20038190.

43. Wang M, Cao R, Zhang L, et al. Remdesivir and chloroquine effectively inhibit the recently emerged novel coronavirus (2019-nCoV) in vitro. Cell Res 2020; 30:269-71.

44. Liu J, Cao R, Xu M, et al. Hydroxychloroquine, a less toxic derivative of chloroquine, is effective in inhibiting SARS-CoV-2 infection in vitro. Cell Discov 2020;6:16.

45. Yao X, Ye F, Zhang M, et al. In vitro antiviral activity and projection of optimized dosing design of hydroxychloroquine for the treatment of severe acute respiratory syndrome coronavirus 2 (SARS-CoV-2). Clin Infect Dis 9 mars 2020. [Cyberpublication avant impression]. pii : ciaa237. doi : 10.1093/cid/ciaa237.

46. Khamitov RA, la Loginova S, Shchukina VN, et al. Antiviral activity of arbidol and its derivatives against the pathogen of severe acute respiratory syndrome in the cell cultures [article en Russe]. Vopr Virusol 2008;53:9-13.

47. Kindler E, Thiel V, Weber F. Interaction of SARS and MERS coronaviruses with the antiviral interferon response. Dans Advances in Virus Research (Ch. 7), dirigé par Ziebuhr, J., Cambridge (Massachusetts) : Academic Press. 2016;96:219-43.

48. Hart BJ, Dyall J, Postnikova E, et al. Interferon- $\beta$ and mycophenolic acid are potent inhibitors of Middle East respiratory syndrome coronavirus in cellbased assays. J Gen Virol 2014;95:571-77.

49. Chan JF, Yao Y, Yeung ML, et al. Treatment with lopinavir/ritonavir or interferon$\beta 1 \mathrm{~b}$ improves outcome of MERS-CoV infection in a nonhuman primate model of common marmoset. J Infect Dis 2015;212:1904-13.

50. Chu CM, Cheng VC, Hung IF, et al.; HKU/UCH SARS Study Group. Role of lopinavir/ ritonavir in the treatment of SARS: initial virological and clinical findings. Thorax 2004;59:252-6

51. Chen F, Chan KH, Jiang J, et al. In vitro susceptibility of 10 clinical isolates of SARS coronavirus to selected antiviral compounds. J Clin Virol 2004;31:69-75.

52. Kalil AC, Treating COVID-19 - Off-label drug use, compassionate use, and randomized clinical trials during pandemics. JAMA 4 mars 2020. [Cyberpublication avant impression]. doi : 10.1001/jama.2020.4742.
Intérêts concurrents : Ning Shen effectue de la recherche dans le cadre d'un essai clinique financé par le Centre des sciences de la santé de l'Université de Pékin portant sur les effets de l'hydroxychloroquine chez les patients atteints de la COVID-19. Aucun autre intérêt concurrent n'a été déclaré.

Cet article a été révisé par des pairs.

Affiliations : Département de pharmacie (W. Liu, P. Zhou, F. Liu, X. Li, N. He, Z. Wu, Q. Zhang, Q. Tang, X. Du, Y. Ying, X. Xu, Y. Zhang, Y. Li, $S$. Zhai), Troisième hôpital universitaire de Pékin, Beijing, Chine; Institut d'évaluation des médicaments (S. Zhai, F. Liu), département d'administration pharmaceutique et de pharmacie clinique ( $Z$. Wu, $X$. Du, Y. Zhang) et département de pneumologie et de médecine de soins intensifs (N. Shen), Centre des sciences de la santé de l'Université de Pékin, Beijing, Chine; Collège de pharmacie (K. Chen), Centre médical de l'Université du Nebraska, Omaha, Nebraska; département des méthodes, des données et de l'incidence de la recherche en santé (Z. Ye, Q. Ibrahim, G. Guyatt), Université McMaster, Hamilton, Ontario; département de pharmacie (X. Gong, J. Liu), Hôpital de Tongji, Collège médical de Tongji, Université des sciences et de la technologie de Huazhong, Wuhan, Hubei, Chine; Institut DeGroote pour la recherche sur la douleur et son traitement (R. Couban), Université McMaster,
Hamilton, Ontario; département de pharmacie (Y. Li), Premier hôpital du Centre hospitalier de Shanxi, Taiyuan, Shanxi, Chine.

Collaborateurs : Wei Liu, Pengxiang Zhou, Ken Chen, Zhikang Ye, Fang Liu, Xiaotong Li, Na He, Ning Shen, Suodi Zhai et Gordon Guyatt ont participé à la conception ou à l'élaboration de l'étude. Wei Liu, Pengxiang Zhou et Ken Chen ont rédigé la première version du manuscrit. Wei Liu, Pengxiang Zhou, Ken Chen, Zhikang Ye, Fang Liu, Xiaotong Li, Na He, Ziyang Wu, Qi Zhang, Xuepeng Gong, Qiyu Tang, Xin Du, Yingqiu Ying, Xiaohan Xu, Yahui Zhang, Jinyu Liu, Yun Li, Ning Shen, Rachel Couban, Quazi Ibrahim, Gordon Guyatt et Suodi Zhai ont recueilli, analysé ou interprété les données. Tous les auteurs ont révisé de façon critique le contenu intellectuel important du manuscrit; ils ont donné leur approbation finale pour la version destinée à être publiée et assument l'entière responsabilité de tous les aspects du travail. Wei Liu, Pengxiang Zhou et Ken Chen ont contribué à ces travaux à parts égales.

Financement : Ken Chen a reçu une bourse du China Scholarship Council.

Partage des données : Toutes les données sont présentées dans les tableaux et les figures de l'article ou de ses annexes.

Accepté : 8 mai 2020

Correspondance : Suodi Zhai, zhaisuodi@163.com 